Supporting Information

\title{
Synthesis and mesomorphism of new alphatic polycarbonates containing side cholesteryl groups
}

Yujiao Xie, Jianshe Hu, Qingwei Dou, Lianhua Xiao, Liqun Yang

Figure S1 ${ }^{1} \mathrm{H}$ NMR spectra of $\mathbf{M}_{\mathbf{L C}}$

Figure S2 ${ }^{1} \mathrm{H}$ NMR spectra of $\mathbf{H P}$

Figure D3 ${ }^{1} \mathrm{H}$ NMR spectra of $\mathbf{C P - 1}$ 